\title{
First-Principles Study of Spin-Orbit Interactions in Bismuth Iron Garnet
}

\author{
Tohru Oikawa $^{1}$, Shugo Suzuki ${ }^{2}$ and Kenji Nakao ${ }^{2}$ \\ ${ }^{1}$ Technical Center, TDK Corporation, Ichikawa, Chiba 272-8558 \\ ${ }^{2}$ Institute of Materials Science, University of Tsukuba, Tsukuba, Ibaraki 305-8573
}

\begin{abstract}
We study the electronic structure of $\mathrm{Bi}_{3} \mathrm{Fe}_{5} \mathrm{O}_{12}$ (BIG) calculated by the fully relativistic first-principles method based on the density functional theory. It is found that the enhancement of the spin-orbit coupling due to the hybridization of Bi $6 p$ is considerably larger in the Fe $3 d$ conduction bands than in the $\mathrm{O} 2 p$ and $\mathrm{Fe} 3 d$ valence bands. The origin of this enhancement is that the Fe $3 d$ conduction bands energetically overlap with Bi $6 p$ bands. The results indicate the significance of spin-orbit coupling in $\mathrm{Fe} 3 d$ conduction bands in relation to the large magneto-optical effect observed in BIG.
\end{abstract}

KEYWORDS: bismuth iron garnet, spin-orbit interaction, LCAO method, fully relativistic band calculation, Faraday rotation

\section{Introduction}

Substitution of $\mathrm{Bi}$ in $\mathrm{Y}$ and rare-earth iron garnets induces the large Faraday rotation (FR). In $\mathrm{Y}_{3-x} \mathrm{Bi}_{x} \mathrm{Fe}_{5} \mathrm{O}_{12}$ (Bi-YIG), the $\mathrm{FR}$ coefficient of $\mathrm{Bi}_{3} \mathrm{Fe}_{5} \mathrm{O}_{12}$ (BIG) increases to about 100 times as large as that of $\mathrm{Y}_{3} \mathrm{Fe}_{5} \mathrm{O}_{12}$ (YIG) at the wavelength of $633 \mathrm{~nm}$. Using this property, these materials are applied to optical isolators, ${ }^{1)}$ which are key devices in a modern optical fiber communication. Although BIG is a thermodynamically nonequilibrium material, it was successfully synthesized as an epitaxial film by Okuda et al. ${ }^{2)}$ Wittekoek et al. ${ }^{3)}$ proposed that the origin of the large $\mathrm{FR}$ is the hybridization of $\mathrm{Bi} 6 p$ orbitals, which possess a large spin-orbit coupling, with the $\mathrm{O} 2 p$ and Fe $3 d$ orbitals, and this idea was also supported by Takeuchi. ${ }^{4)}$ By taking the magnitude of the spin-orbit coupling as a parameter, Shinagawa, ${ }^{5)}$ and Zenkov and Moskvin ${ }^{6}$ ) have explained the FR spectra of Bi-YIG in the framework of the cluster molecular orbital theory. In their analysis, the enhancement of spin-orbit coupling of $\mathrm{Fe} 3 d$ orbitals was assumed to be much smaller than that of $\mathrm{O} 2 p$ orbitals, because Fe sites are located more distant than $\mathrm{O}$ sites from $\mathrm{Bi}$ sites, and so the hybridization is possibly reduced with overlap integrals.

Although the affection of $\mathrm{Bi}$ on spin-orbit interaction has drawn a great interest, no theoretical studies have been carried out based on the band structures to justify the previous analysis. The most serious difficulty in performing band structure calculations would be that the garnet structure is so complex and contains 80 atoms in a bcc primitive unit cell, which makes the calculations quite formidable. Recently, band structure calculatios of YIG have been carried out for the first time ${ }^{7,8)}$ within a scalar relativistic treatment, but the band 
structure of BIG is not known yet.

In this paper, we report the results of the fully relativistic first-principles calculations on the electronic structures of BIG and YIG. The change of spin-orbit interaction due to $\mathrm{Bi}$ is directly investigated from the one-electron energy level. The method of calculation is given in the next section. The results of the geometry optimization and the band structure calculations are presented in $\S 3$, together with discussions. Conclusion is given in $\S 4$.

\section{Method of Calculation}

We employ a full-potential linear-combination-of-atomic-orbitals (LCAO) method based on the density functional theory within the local-spin-density-approximation (LSDA). The used exchange-correlation potential is the Perdue-Zunger parametrization ${ }^{9}$ ) of the results of the numerical study by Ceperley and Alder. ${ }^{10)}$ To distinguish the effect of spin-orbit coupling, two types of relativistic calculations are carried out; one is the fully relativistic full potential LCAO (FFLCAO) calculations ${ }^{11)}$ and the other is the scalar relativistic full potential LCAO (SFLCAO) calculations, ${ }^{12}$ ) where the former includes all the relativistic effects while the latter includes relativistic effects except the spin-orbit coupling. In the FFLCAO calculations, we solve the Dirac-Kohn-Sham equations directly by adopting the atomic orbitals of four component Dirac spinors with positive energy as basis functions. In the SFLCAO calculations, we solve a scalar relativistic Korn-Sham equations which takes the scalar relativistic effects into account as the increase in the mass of electrons due to the relativistic effects. The details of the relativistic formulation employed in the present study are found in refs. 11 and 12 .

The geometry optimization is carried out by calculating the forces acting on the atoms and searching the atomic coordinates of the equilibrium geometry; we calculate the Hellman-Feynman forces and Pulay forces by using 4 special k points in the full Brillouin zone. As basis functions, we take account of all electrons including core electrons, adopting not only atomic orbitals of neutral atoms but also those of positively charged atoms to describe the contraction of atomic orbitals in solids. The used atomic orbitals are [Xe]; $4 f, 5 d, 6 s, 6 p$ (neutral); $6 s, 6 p, 6 d(2+)$ for $\mathrm{Bi},[\mathrm{Kr}] ; 4 d, 5 s$ (neutral); $5 p(1+) ; 4 d, 5 s(2+) ; 5 p(3+)$ for $\mathrm{Y},[\mathrm{Ar}] ; 3 d, 4 s$ (neutral); $4 p(1+) ; 3 d, 4 s(2+) ; 4 p(3+)$ for $\mathrm{Fe}$, and $1 s, 2 s, 2 p$ (neutral); $2 s, 2 p(2+) ; 3 d(6+)$ for $\mathrm{O}$, where square brackets denote atomic orbitals of the rare gas closed shells and the valence of positively charged atoms are shown in parentheses.

\section{Results and Discussions}

\subsection{Geometry optimization}

We optimize the geometries of BIG and YIG by the FFLCAO calculations, fixing the lattice constants as the experimental values, $12.624 \AA$ for BIG and $12.376 \AA$ for YIG, and changing the oxygen parameters, $x, y$, and $z$; the space group of garnet structure is $I a 3 d$ or $O_{h}^{10}$, where the atomic coordinates of cations are uniquely determined by the lattice constant 
while the atomic coordinates of oxygen atoms possess three degrees of freedom, $x, y$, and $z$. As a result, we obtain $x=-0.0314, y=0.0512$, and $z=0.1480$ for BIG and $x=-0.0281, y=$ 0.0554 , and $z=0.1506$ for YIG. It is established empirically that the oxygen parameters can be approximated by a linear expression of the ionic radii of cations and this expression is extended to the ferrimagnetic garnets by Hawthorne. ${ }^{13)}$ In Table I, we show the distances between the cations and the oxygens in BIG and YIG using the optimized oxygen parameters with those obtained by the Hawthorne's expression and, for comparison, also with the experimental ones of YIG; $x=-0.0275, y=0.0572$, and $z=0.1495 .{ }^{14)}$ The results show a good agreement, where the discrepancy is mostly less than $1 \%$, and thus the geometry optimization can be justified.

\subsection{Band structure}

The band structures of BIG calculated at the optimized geometry by the SFLCAO and the FFLCAO calculations are shown in Fig. 1(a) and (b), respectively, where the up-spin and down-spin bands are shown together in Fig. 1 (a) and the origin of energy is taken at the top of the valence bands. Both results show that BIG is a ferrimagnetic insulator with a direct gap at $\Gamma$ point, where up-spin Fe $3 d$ atomic orbitals at 24 d sites and down-spin Fe $3 d$ atomic orbitals at $16 \mathrm{a}$ sites are occupied. However, due to LSDA, each Fe $3 d$ magnetic moment is shrunk to about $70 \%$ compared to the ideal spin of $5 / 2$. The calculated band gaps are 0.45 $\mathrm{eV}$ and $0.33 \mathrm{eV}$ for the SFLCAO and the FFLCAO results, respectively. In case of YIG, the calculated band gaps are $0.31 \mathrm{eV}$ and $0.30 \mathrm{eV}$ for the SFLCAO and the FFLCAO results, respectively, and these magnitude are considerably smaller than the experimental one of 2.85 $\mathrm{eV}^{15)}$ estimated by the temperature dependence of the electric resistivity. The underestimation of the band gap is likely attributed to the LSDA.

We next show the calculated total density of states (DOS) and the partial density of states (PDOS) of BIG in Fig. 2 (a) and (b) obtained by the SFLCAO and the FFLCAO calculations, respectively. The PDOS are shown for Fe $3 d$, O $2 p$, and $\mathrm{Bi} 6 p$, which are the main components near the band gap. The PDOS of Bi $6 p$ by the FFLCAO calculations are decomposed into the

Table I. Distance between cations and oxygen atoms in $\AA$.

\begin{tabular}{lccccc}
\hline & \multicolumn{2}{c}{ BIG } & \multicolumn{3}{c}{ YIG } \\
& Calc. & Hawthorne & Calc. & Hawthorne & Expt. \\
\hline Fe-O (16a) & 2.016 & 2.035 & 2.016 & 2.018 & 2.010 \\
Fe-O (24d) & 1.863 & 1.867 & 1.850 & 1.867 & 1.872 \\
Bi,Y-O (1) & 2.444 & 2.440 & 2.361 & 2.354 & 2.369 \\
Bi,Y-O (2) & 2.557 & 2.533 & 2.454 & 2.434 & 2.429 \\
\hline
\end{tabular}




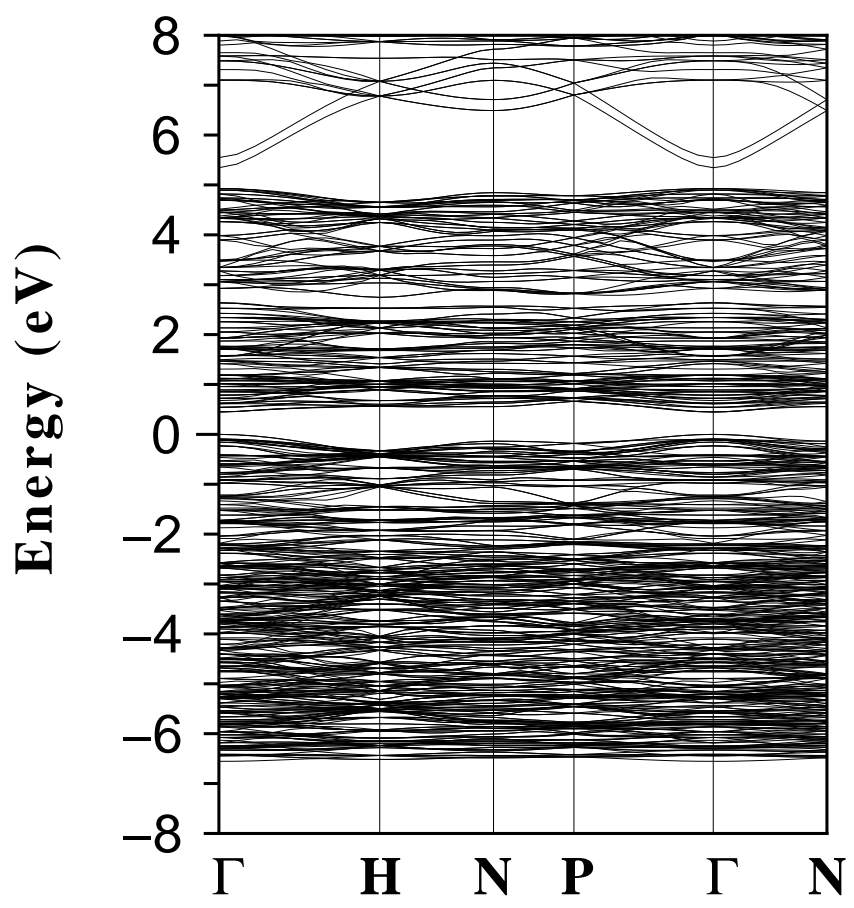

(a)

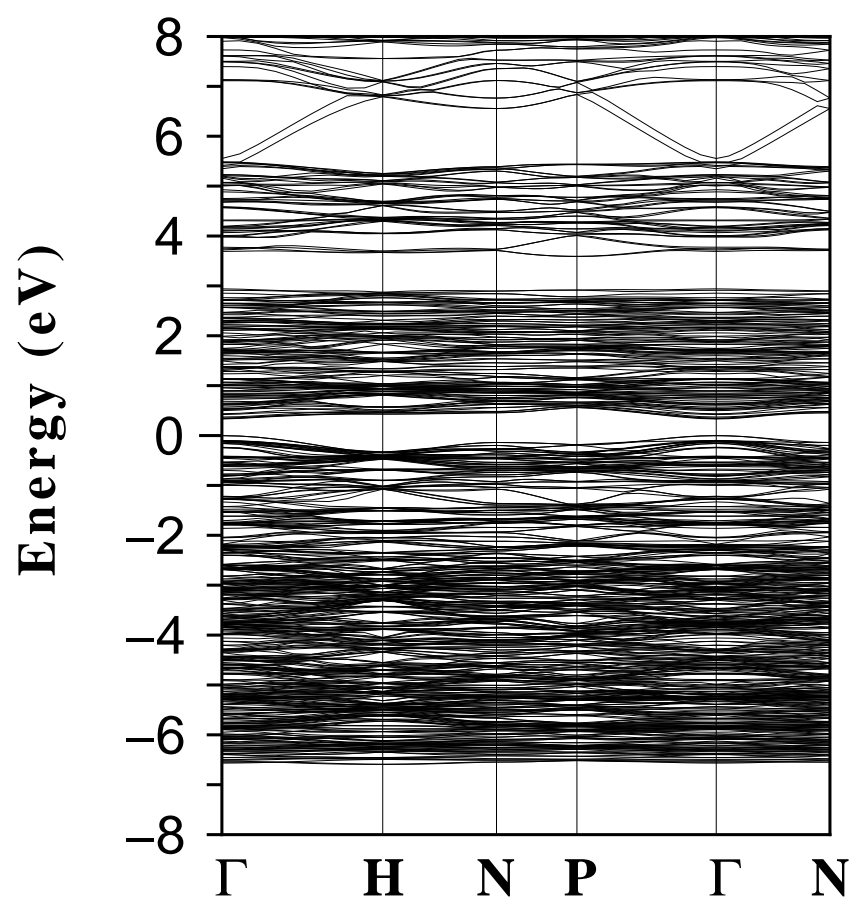

(b)

Fig. 1. Band structures of BIG by (a) SFLCAO calculations and (b) FFLCAO calculations.

total angular momenta $\mathrm{J}$ and the spin directions. In both of the SFLCAO and the FFLCAO results, the width of the valence band is $6.5 \mathrm{eV}$ and its main characters are Fe $3 d$ and $\mathrm{O} 2 p$; the contribution of $\mathrm{O} 2 p$ is larger than Fe $3 d$ below $-2 \mathrm{eV}$ while the contribution of $\mathrm{Fe} 3 d$ is larger than $\mathrm{O} 2 p$ above $-2 \mathrm{eV}$. On the other hand, the lower energy part of conduction band is 
mainly composed of Fe $3 d$ and the higher energy part is mainly composed of $\mathrm{Bi} 6 p$, where they are separated by the small gap of $0.2 \mathrm{eV}$ in the SFLCAO results. When spin-orbit coupling is taken into account, the $\mathrm{Bi} 6 p$ bands are splitted into the $\mathrm{J}=3 / 2\left(6 p_{3 / 2}\right)$ and $\mathrm{J}=1 / 2\left(6 p_{1 / 2}\right)$ components, and the latter bands overlap on the upper edge of the $\mathrm{Fe} 3 d$ bands, generating the energy gap around at $3.5 \mathrm{eV}$. The magnitude of the spin-orbit splitting is about $2 \mathrm{eV}$, which is in good agreement with the experimental observation of $1.7 \mathrm{eV}$ obtained for $\mathrm{Bi}_{2} \mathrm{CuO}_{4}{ }^{16}$ ) and $2.16 \mathrm{eV}$ for solid Bi. ${ }^{17}$ )

We also obtain the similar PDOS of YIG for the valence band and the lower conduction band, and it is found that the Y $4 d$ band forms the higher conduction band, which is consistent with the results of refs. 7 and 8. In contrast to the BIG results, there are no noticeable band splittings due to spin-orbit coupling in Y $4 d$ orbitals. The details of the results on YIG will be published elsewhere.

\subsection{Evaluation of spin-orbit interaction}

Based on the calculated band structures, we estimate the change of spin-orbit coupling due to Bi. Unlike in the case of Bi $6 p$ bands, it is difficult to determine the splittings of $\mathrm{Fe}$ $3 d$ or $\mathrm{O} 2 p$ bands from the PDOS because the splittings are much smaller than their band width. By investigating the band degeneracy, we notice that the degree of degeneracy is reduced at every $\mathrm{k}$ point due to spin-orbit coupling, and especially at $\Gamma$ point it is found that the degeneracy is completely removed while there exists the degeneracy of up to threefold in SFLCAO results. Using this characteristic, we assign each band in FFLCAO results to the corresponding SFLCAO band at $\Gamma$ point, then we estimate the magnitude of spin-orbit coupling by defining the level splitting as the difference of two energies for each doubly degenerated SFLCAO bands and also as the half of the difference between the highest and the lowest energies for each triply degenerated SFLCAO bands.

In Fig. 3 (a), (b) and (c), we show the magnitude of the level splittings defined above as a function of energy. Those figures represent the upper part of $\mathrm{O} 2 p$ valence bands, the upper part of $\mathrm{Fe} 3 d$ valence bands, and the lower part of $\mathrm{Fe} 3 d$ conduction bands, respectively. In the $\mathrm{O} 2 p$ valence bands of BIG, the largest splitting is $61.5 \mathrm{meV}$ and the average is $10.2 \mathrm{meV}$ and, in those of YIG, the former is $8.8 \mathrm{meV}$ and the latter is $3.2 \mathrm{meV}$. In the Fe $3 d$ valence bands of BIG, the largest splitting is $24.4 \mathrm{meV}$ and the average is $8.0 \mathrm{meV}$ and, in those of YIG, the former is $8.1 \mathrm{meV}$ and the latter is $3.7 \mathrm{meV}$. In the Fe $3 d$ conduction bands of BIG, the largest splitting is $44.9 \mathrm{meV}$ and the average is $22.1 \mathrm{meV}$ and, in those of YIG, the former is $19.7 \mathrm{meV}$ and the latter is $9.1 \mathrm{meV}$. Taking the average values, it is found that Bi causes level splittings most strongly in the Fe $3 d$ conduction bands; the reason is that Bi $6 p$ bands energetically overlap with Fe $3 d$ conduction bands.

We now compare the calculated spin-orbit coupling with the experimental one. Because one-electron spin-orbit coupling in the excited states of free $\mathrm{Fe}^{3+}$ ion is experimentally ob- 

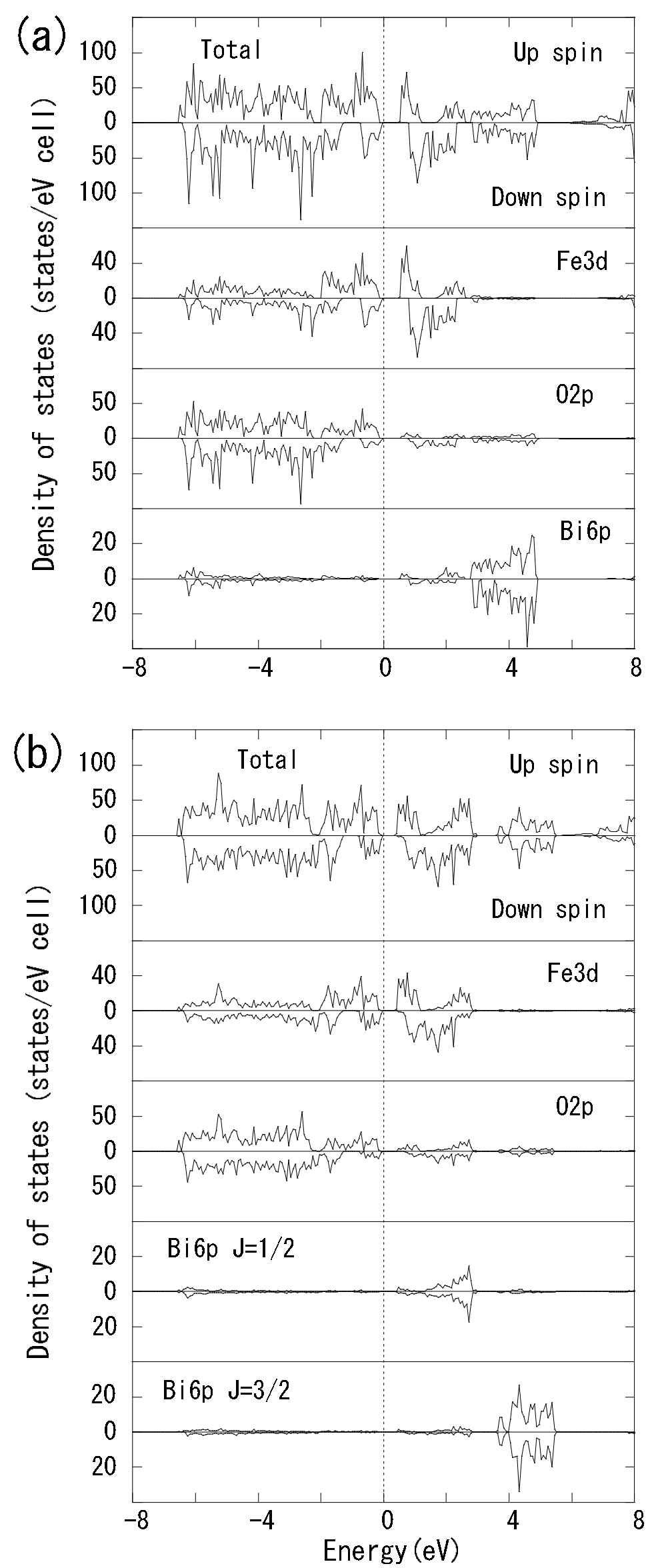

Fig. 2. The total and partial DOS of BIG by (a) $\$$ PCAO calculations and (b) FFLCAO calculations. 


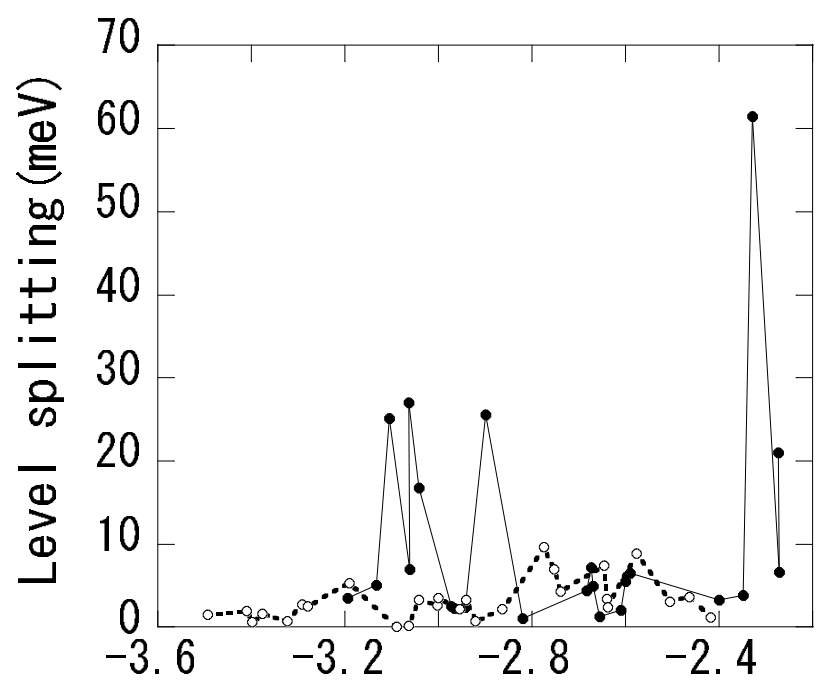

(a)

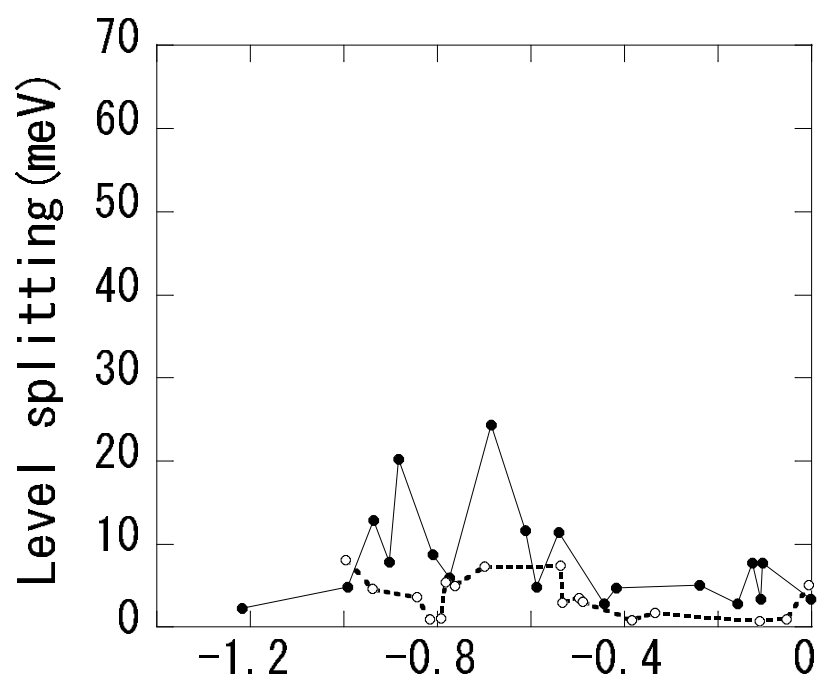

(b)

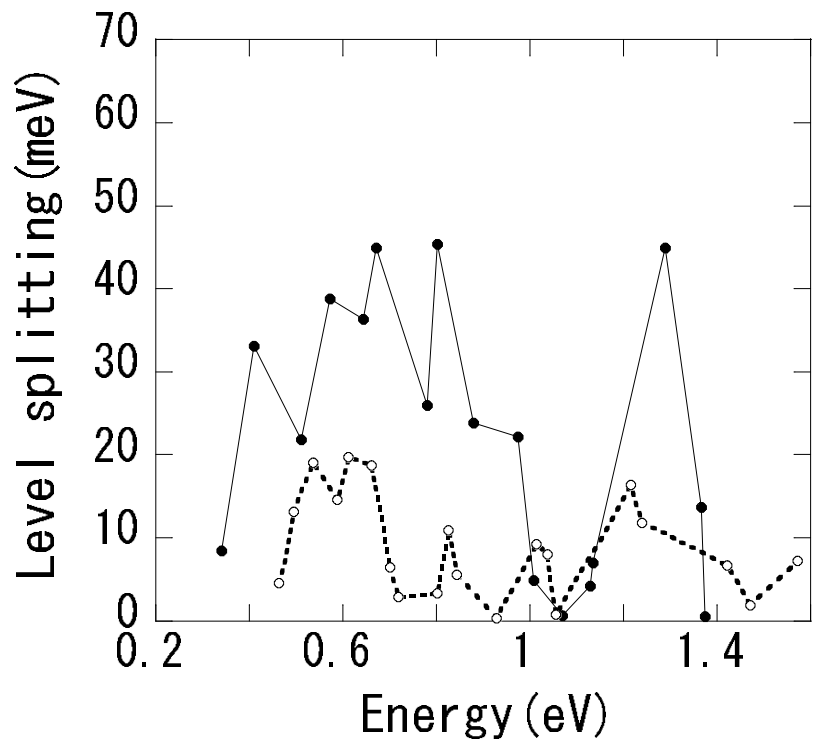

(c)

Fig. 3. Level splittings of BIG (solid line) and YIG (dotted line) in (a) the upper part of O $2 p$ valence bands, (b) the upper part of $\mathrm{Fe} 3 d$ valence bands, and (c) the lower part of $\mathrm{Fe} 3 d$ conduction bands. The level splitting is defined as the difference of two energies for doubly degenerated states, and as the half of the difference between the highest and the lowest energies for triply degenerated states. 
served to be $52 \mathrm{meV}{ }^{18)}$ the effective spin-orbit coupling for the $t_{2 g}$ or $t_{2}$ states, which are the octahedral or tetrahedral crystal field levels of triplet, are also estimated to be at most $52 \mathrm{meV}$. In BIG and YIG, the $\mathrm{t}_{2 g}$ and $\mathrm{t}_{2}$ states contribute to the spin-orbit coupling in the $\mathrm{Fe} 3 d$ bands. So, the value to be compared is $39.4 \mathrm{mV}$, which is the twice of the largest level splitting in Fe $3 d$ conduction bands of YIG mentioned above. As a moderate magnitude in YIG, it was estimated to be $44 \mathrm{meV}$ by Wittekoek et al. considering the reduction due to the covalency of Fe $3 d$ orbitals in solids. This value is quite near to the calculated one. As for BIG, in the same manner as YIG, spin-orbit coupling to be compared is $89.8 \mathrm{meV}$. This value is too large to be attributed only to spin-orbit coupling emerging from the free $\mathrm{Fe}^{3+}$ ion, and surely contains contribution of Bi.

From the Mulliken population analysis, the average hybridization of the $\mathrm{Bi} 6 p$ orbitals at $\Gamma$ point are found to be $3.9 \%$ for the O $2 p$ valence bands, $3.1 \%$ for the Fe $3 d$ valence bands, and $5.9 \%$ for the $\mathrm{Fe} 3 d$ conduction bands. We can find the clear correlation between these values and the aforementioned level splittings due to Bi. It is also obvious that the enhancement of level splittings due to $\mathrm{Bi}$ in the $\mathrm{Fe} 3 d$ conduction bands is obtained for most of the bands. On the other hand, the enhancement in the $\mathrm{O} 2 p$ valence bands is obtained only for several specific bands.

Finally, we refer to the significance of the present results in relation to the FR of BIG. It is experimentally observed that the most important contribution to the FR induced by $\mathrm{Bi}$ in the visible wavelength region comes from the optical excitation within $1 \mathrm{eV}$ from the optical absorption edge. ${ }^{3)}$ As shown in the PDOS of BIG within the energy range of $1 \mathrm{eV}$ from the bottom of the conduction bands, Fe $3 d$ character is predominant and the $\mathrm{Bi} 6 p$ component is only several \%. Therefore, Bi contributes to the FR not through the direct electronic transition to Bi $6 p$ bands, but through the hybridization with $\mathrm{O} 2 p$ and $\mathrm{Fe} 3 d$ bands. Thus we confirm the basic assumption made by Wittekoek et al. Contrary to the earlier studies, however, our results show that the enhancement of spin-orbit coupling in the Fe $3 d$ conduction bands is larger than that in the $\mathrm{O} 2 p$ and $\mathrm{Fe} 3 d$ valence bands because of the band overlapping with $\mathrm{Bi}$ $6 p$ bands. It implies that this band overlapping may play an essential role to induce the large FR.

\section{Conclusion}

In the present study, we have performed the band structure calculations of BIG and YIG by the fully relativistic and the scalar relativistic treatments. As a geometry optimization, the oxygen parameters of BIG have been optimized, and the obtained cation-oxygen distances show a good agreement with those obtained from the Hawthorne's expression. By comparing the band degeneracy between the two relativistic calculations, it was found that the degree of degeneracy in the scalar relativistic treatment is reduced at every $\mathrm{k}$ point due to spin-orbit coupling, and it dissolves into singlet completly at $\Gamma$ point. Therefore, the energy splittings 
at $\Gamma$ point have been evaluated as a magnitude of spin-orbit interaction. As a main result, we found that the enhancement of spin-orbit coupling due to $\mathrm{Bi}$ is larger in the Fe $3 d$ conduction bands than in the $\mathrm{O} 2 p$ and $\mathrm{Fe} 3 d$ valence bands. This finding is consistet with the magnitude of hybridization of Bi $6 p$ orbitals with those bands, since the Fe $3 d$ conduction bands energetically overlap with the $\mathrm{Bi} 6 p$ bands. This result differs from the earlier observation, which predicts that the largest enhancement will appear in the $\mathrm{O} 2 p$ bands because $\mathrm{O}$ atoms are the nearest to $\mathrm{Bi}$ atom. The large FR might be related to the band overlapping between Fe $3 d$ conduction bands and Bi $6 p$ bands. Clarifying this point will be our future work.

\section{Acknowledgement}

The authors are grateful to Prof. K. Shinagawa of Toho University for valuable discussions. 


\section{References}

1) T. Hibiya, T. Ishikawa and Y. Ohta: IEEE Trans. Magn. MAG-22 (1986) 11.

2) T. Okuda, T. Katayama, H. Kobayashi, N. Kobayashi, K. Satoh and H. Yamamoto: J. Appl. Phys. 67 (1990) 4944.

3) S. Wittekoek, T. J. A. Popma, J. M. Robertson and P. F. Bongers: Phys. Rev. B 12 (1975) 2777.

4) H. Takeuchi: Jpn. J. Appl. Phys. 14 (1975) 1903.

5) K. Shinagawa: Magneto-Optics, ed. S. Sugano and N. Kojima (Springer, Berlin, 2000), chap.5, p.137.

6) A. Z. Zenkov and A. S. Moskvin: J. Phys.: Condens.Matter 14 (2002) 6957.

7) Y.-N. Xu, Z.-Q. Gu and W. Y. Ching: J. Appl. Phys. 87 (2000) 4867.

8) W. Y. Ching, Z.-Q. Gu and Y.-N. Xu: J. Appl. Phys. 89 (2001) 6883.

9) J. P. Perdew and A. Zunger: Phys. Rev. B 23 (1981) 5048.

10) D. M. Ceperley and B. J. Alder: Phys. Rev. Lett. 45 (1980) 566.

11) S. Suzuki and K. Nakao: J. Phys. Soc. Jpn. 68 (1999) 1982.

12) S. Suzuki and K. Nakao: J. Phys. Soc. Jpn. 69 (2000) 532.

13) F. C. Hawthorne: J. Solid State Chem. 37 (1981) 157.

14) S. Geller: Z. Krist. 125 (1967) 1.

15) R. Metselaar and P. K. Larsen: Solid State Commun. 15 (1974) 291.

16) A. Goldoni, U. D. Pennino, F. Parmigiani and A. Revcolevschi: Solid State Commun. 90 (1994) 161.

17) L. Ley, R. Pollak, S. Kowalczyk and D. A. Shirley: Phys. Letters 41A (1972) 429.

18) J. J. Krebs and W. G. Maisch: Phys. Rev. B 4 (1971) 757. 